\title{
Unusual Karyotype in Acute Myelomonocitic Leukemia: A Case Report
}

\author{
MARIA LETIZIA CONSOLI ${ }^{1}$, ALESSANDRA ROMANO $^{1}$, NUNZIATINA LAURA PARRINELLO $^{1}$, \\ LOREDANA TAMBÈ ${ }^{1}$, MARIA ANNA ROMEO ${ }^{1}$, DOMENICO SALEMI ${ }^{2}$, \\ ALESSANDRA SANTORO $^{2}$ and FRANCESCO DI RAIMONDO ${ }^{1}$

\begin{abstract}
${ }^{1}$ Division of Hematology and Bone Marrow Transplant, A.O.U. Policlinico Vittorio Emanuele, Catania, Italy; ${ }^{2}$ Division of Hematology and Bone Marrow Transplant, A.O. Ospedali Riuniti Villa Sofia-Cervello, Palermo, Italy
\end{abstract}

\begin{abstract}
Background/Aim: Acute myeloid leukemia is well characterized by chromosomal aberrations that correspond to various subtypes of acute leukemias. The $t(8 ; 21)(q 22 ; q 22)$ is a frequent chromosomal abnormality strongly associated with acute myeloblastic leukemia with maturation (AML-M2), but is rarely associated with other subtypes. Translocation involving a third chromosome could produce new genetic rearrangements that lead to leukemogenesis. Patients and Methods: Conventional cytogenetic analysis and fluorescence in situ hybridization (FISH) were performed to identify the karyotype. Reverse transcriptase polymerase chain reaction (RT-PCR) was used to detect the AML1/ETO transcript. Results/Conclusion: We herein report a novel rearrangement with a three-way translocation involving chromosomes 8, 21 and another unknown chromosome, in an 83-year-old female patient diagnosed as AML-M4, with an ALM1/ETO negative transcript. This is an uncommon case of AML-M4 with threeway translocation in a new variant of $t(8 ; 21)$ acute myeloid leukaemia. The detailed mechanism of different phenotype expression is unclear. Further study is needed to identify the leukemogenetic transformation resulting from $t(8 ; 21)$ translocation.
\end{abstract}

Acute myeloid leukaemia (AML) is a heterogeneous disease. Chromosome translocations are common genetic abnormalities in leukaemia and may contribute to leukemogenesis. Their definition is of paramount importance since it has been demonstrated that the karyotype is one of the most important prognostic factors. The $t(8 ; 21)(\mathrm{q} 22 ; \mathrm{q} 22)$ translocation is a frequent non-random cytogenetic

Correspondence to: Maria Letizia Consoli, Division of Hematology and Bone Marrow Transplant, A.O.U. Policlinico Vittorio Emanuele, Catania, Italy. Via Santa Sofia, 78, Catania, 95123, Italy. Tel: +39 0953781988, Fax: +39 0953781956, e-mail: marzia.consoli@libero.it

Key Words: AML, variant $\mathrm{t}(8 ; 21)$, three-way translocation. abnormality in AML that is responsible for the fusion of two genes, the AML1 (RUNX1) and ETO (RUNX1T1), giving rise to the AML1/ETO fusion gene that identifies a type of AML defined as AML-M2 in the French-American-British (FAB) classification systems, a leukemia with a minimal or absent monocytic component (1). However, this translocation is not exclusively observed in M2 AML since different rearrangements of the chromosome band 21q22 can occur more rarely in other human malignant blood disorders such as M1 AML involving the AML1 gene (2). In addition, complex variant translocations involving 8q22, 21q22, and another chromosome can occur with a frequency of $3.4 \%$ (3). AML1 may have several partners resulting in gene fusions in different chromosomal translocations (4-5), but some of these rearrangements do not result in chimeric genes and modify the functions of the truncated $A M L 1$ gene. Herein, we report a three-way translocation involving chromosomes 8, 21 and another unknown one, in an AML1/ETO negative patient.

\section{Patients and Methods}

An 83-year-old female was admitted in the Division of Haematology, A.O.U. Policlinico-Vittorio Emanuele (Catania) in October 2017 complaining of extensive spontaneous ecchymosis. Hematologic evaluation showed absolute leukocyte count of $7,810 \times 10^{9} / 1$, platelet count of $10,000 \times 10^{9} / 1$, haemoglobin concentration of $10.7 \mathrm{~g} / \mathrm{dl}$, associated to slight reduction of B12 vitamin dosage. Flow cytometry with five-colour analysis using monoclonal antibodies was performed on a bone marrow sample revealing the co-existence of $25.7 \%$ of myeloid blast cells and $20.4 \%$ of monocytes. Myeloblasts co-expressed the following markers: CD33, CD38, HLA-DR, CD45RA, MPO, CD45 and were positive for the CD34 and CD117 immaturity markers. The monocyte component was negative for CD34 and CD117 and positive for CD4, CD33, CD36, CD38, HLA-DR, Lys, MPO and CD45. The immunophenotypic investigation and the observation of the morphological preparation was compatible with the diagnosis of AML with a monocyte component (6). Molecular profiling identified a wild-type Fms related tyrosine kinase 3-Internal tandem duplication (FLT3-ITD) and Nucleophosmin (NPM). Conventional 
cytogenetic analysis on bone marrow cells at the time of diagnosis showed: 46, XX, t(8;21)(q22;q22) in 95\% of metaphases (19/20) (Figure 1a), and 46, XX in 5\% of metaphases (1/20). The transcript AML1/ETO was checked by reverse transcriptase polymerase chain reaction (RT-PCR) and the results were negative. Fluorescence in situ hybridization (FISH) analysis on interphase nuclei (Figure 1b) showed three green signals for the $A M L 1$ gene (locus 21q22) and two red signals for the ETO gene (locus 8q22), but no fusion signal. These data together confirmed a rearrangement of the AML1 gene but not a rearrangement of the ETO gene and the involvement of a third unidentified chromosome. Due to patient's age and the presence of co-morbidities, the patient was given azacytidine, 100 mg flat dose, on days 1-5 every 28 days, but treatment was stopped earlier for supervening pneumonia. The patient died in December 2017, two months after diagnosis.

Ethics approval and informed consent. The patient provided written informed consent to diagnostic procedures. The study adheres to the declaration of Helsinki and the biological samples were collected following an institutionally approved protocol at the Azienda Ospedaliero-Universitaria "Policlinico-Vittorio Emanuele", Catania, Italy.

\section{Results}

Here, we report a case of AML-M4 with $\mathrm{t}(8 ; ?, 21)(\mathrm{q} ? ;$;,q22) translocation and AML1/ETO negative transcript. This case confirms that conventional cytogenetics and FISH analyses are still necessary for the determination of the genomic abnormalities that characterize AML. Evaluation of AML1ETO by PCR as well as the search for NPM and FLT3-ITD mutations, were not informative in this case, indicating that molecular biology findings should always be considered together with all the other clinical and laboratory informations and should not be considered as substitutes of conventional cytogenetics. Most of the cases with the $\mathrm{t}(8 ; 21)(\mathrm{q} 22 ; \mathrm{q} 22)$ translocation are classified as AML-M2. The finding of $\mathrm{t}(8 ; 21)$ has been reported infrequently in other FAB sub-types of AML (M1 and M4) (7), myelodysplastic syndrome (8), and the blastic phase of chronic myelogenous leukemia (9). This translocation is responsible for the AML1/ETO fusion gene that is considered one of the most common genetic rearrangements that drives the development of AML. However, complex variants of $\mathrm{t}(8 ; 21)$ are occasionally observed in a small percentage of AMLs (10). Most are three-way translocations involving chromosomes 8 , 21 and a third chromosome such as $1,2,3,5,6,8,10,12$, $13,14,15,16,17,18,20$ and $\mathrm{X}(11-14)$.

\section{Discussion}

We report a new translocation involving the $A M L 1$ gene in an 83-year-old patient with AML-M4. Analysis of a conventional karyotype showed 46, XX, t(8;21)(q22;q22) [19]/46, XX[1], but FISH on interphase nuclei and metaphases showed the involvement of an unknown third chromosome. The main question arising by the detection of a novel translocation involving the $A M L 1$ rearrangement is whether it results in a fusion gene with a gene located in the partner chromosome of the translocation different from ETO, or if the truncation of the AML1 gene per se is sufficient to cause leukemia. The two possibilities have been reported in the literature (15). The relationship between the variations of genes like $A M L 1$, the genesis of the different subtypes of leukemia and their evolution is known, but the detailed mechanism of the different phenotypes is not clear and reinforces the idea that this region could be an important gene in the pathogenesis of AML. In order to understand the molecular mechanism involved in unusual rearrangements and the significance of this additional information $(16,17)$, evaluation of the single nucleotide polymorphism (SNP) profiling and loss of heterozygosity regions $(\mathrm{LOH})$ is necessary $(18,19)$. It could help to more precisely define the genomic alterations that can reflect clinical features and prognosis.

\section{Conflicts of Interest}

The Authors declare that they have no competing interests regarding this study.

\section{Author's Contributions}

MLC drafted the manuscript; MLC and AR were responsible of study concept; MLC, LT, NLP, AS and DS performed molecular, cytogenetic and immunophenotypic analyses; MLC analysed and interpreted the data; AR and MAR made a critical revision of the paper and managed the patient; FDR conceived the original idea and supervised the project.

\section{Acknowledgements}

Funding: Maria Letizia Consoli received funding from Regione Sicilia, Piano Sanitario Nazionale 2014, Linea Progettuale 6-Azione 6.13 .

\section{References}

1 Erickson P, Gao J, Chang KS, Look T, Whisenant E, Raimondi $\mathrm{S}$, Lasher R, Trujillo J, Rowley J and Drabkin H: Identification of breakpoints in $\mathrm{t}(8 ; 21)$ acute myelogenous leukemia and isolation of a fusion transcript, AML1/ETO, with similarity to Drosophila segmentation gene, runt. Blood 80(7): 1825-1831, 1992. PMID: 1391946.

2 Gamou T, Kitamura E, Hosada F, Shimizu K, Shinohara K, Hayshi Y, Nagase T, Yokoyama Y and Ohki M: The partner gene of AML1 in $\mathrm{t}(16 ; 21)$ myeloid malignancies is a novel member of the MTG8(ETO) family. Blood 91(11): 4028-4037, 1998. PMID: 9596646.

3 Groupe Français de Cytogénétique Hématologique: Acute myelogenous leukemia with an 8;21 translocation: A report on 148 cases from the Groupe Français de Cytogénétique Hématologique. Cancer Genet Cytogenet 44(2): 169-179, 1990. PMID: 2297675. 
a

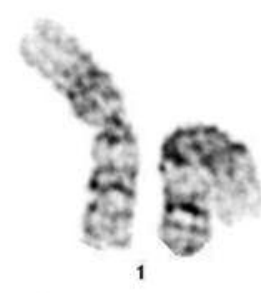

Top

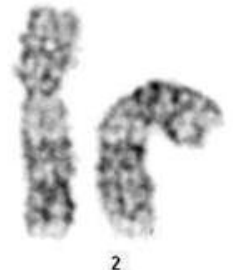

2

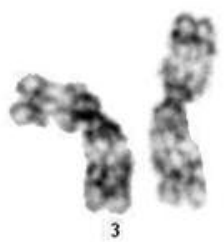

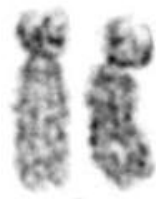

4

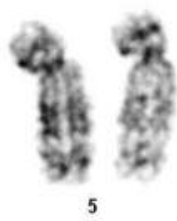

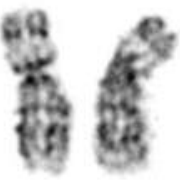

6

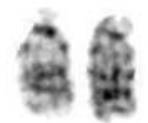

13

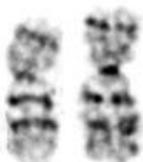

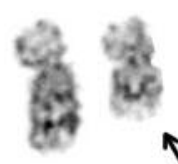

8

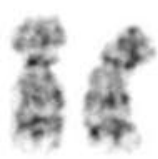

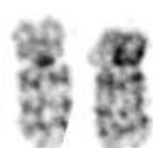

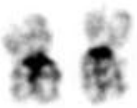

16

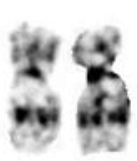

11

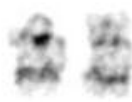

17

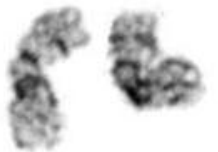

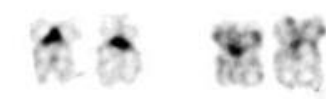
$19 \quad 20$

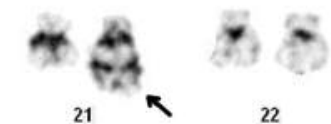
$x$

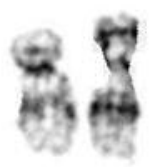

12

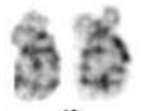

18

b

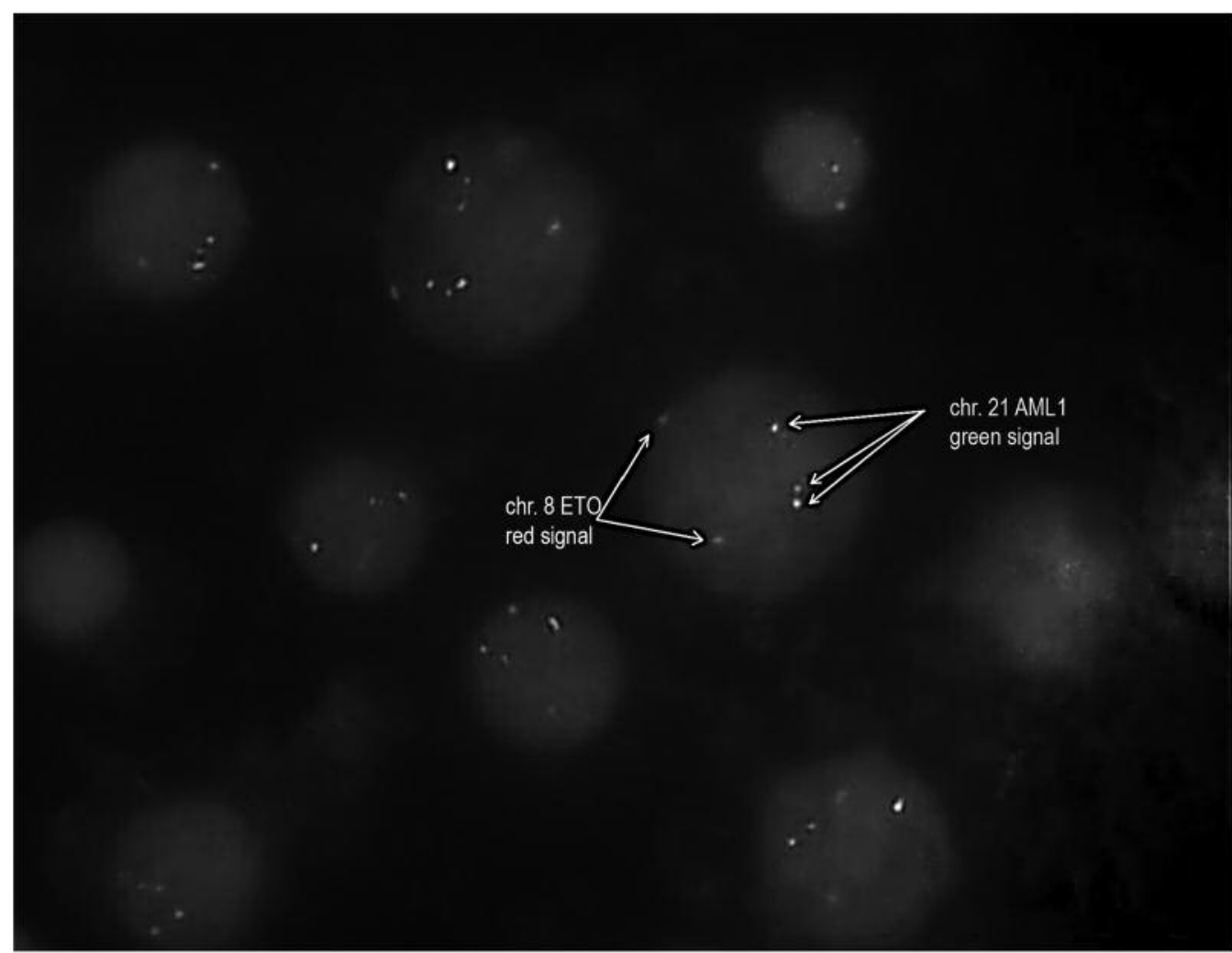

Figure 1. Conventional cytogenetic and FISH analyses of AML-M4 with $t(8 ; ?, 21)(q ? ; ?, q 22)$. a. G-banded karyogram of 19/20 marrow cells metaphases. Arrows indicate the t(8;21) translocation. b. FISH using AML1/ETO dual color, dual fusion probe showed three (green) signals (AML1, locus 21q22) and two (red) signals (ETO, locus 8q22) but no AML1-ETO fusion signal was detected confirming a rearrangement of AML1, but not a rearrangement of ETO and the involvement of a third unidentified chromosome. 
4 Vunditi BR, Kerketta L, Madkaikar M, Jijina F and Ghosh K: Three way translocation in a new variant of $\mathrm{t}(8 ; 21)$ acute myeloid leukemias involving Xp22. Indian J Cancer 45(1): 3033, 2008. PMID: 18453738 .

5 Udayakumar AM, Alkindi S, Pathare AV and Raeburn JA: Complex $\mathrm{t}(8 ; 13 ; 21)(\mathrm{q} 22 ; \mathrm{q} 14 ; \mathrm{q} 22)$ - a novel variant of $\mathrm{t}(8 ; 21)$ in a patient with acute myeloid leukemia (AMLM2). Arch Med Res 39(2): 252-256, 2008. PMID: 18164974. DOI: 10.1016/j.arcmed. 2007.09.002

6 Shang L, Chen X, Liu Y, Cai X, Shi Y, Shi L, Li Y, Song Z, Zheng B, Sun W, Ru K, Mi Y, Wang J and Wang H: The immunophenotypic characteristics and flow cytometric scoring system of acute myeloid leukemia with $\mathrm{t}(8 ; 21)$ (q22; 22$)$; RUNX1-RUNX1T1. Int J Lab Hematol 41(1): 23-31, 2019. PMID: 30264491. DOI: 10.1111/ijlh.12916

7 Kwong YL, Ching LM, Liu HM, Lee CP, Pollock A and Chan LC: $8 ; 21$ translocation and multilineage involvement. Am J Hematol 43(3): 212-216, 1993. PMID: 8352238.

8 Taj AS, Ross FM, Vickers M, Choudhury DN, Harvey JF, Barber JC, Barton C and Smith AG: $\mathrm{t}(8 ; 21)$ myelodysplasia, an early presentation of M2 AML. Br J Haematol 89(4): 890-892, 1995. PMID: 7772527.

9 Ferro MT, Steegman JL, Escribano L, Heiurichs B, Parada L, García-Sagredo JM, Resino M, Cabello P and San Román C: Phpositive chronic myeloid leukemia with $\mathrm{t}(8 ; 21)(\mathrm{q} 22 ; \mathrm{q} 22)$ in blastic crisis. Cancer Gen Cytogen 58(1): 96-99, 1992. PMID: 1728959.

10 Gmidène A, Sennana H, Frikha R, Elloumi M, Belaaj H and Saad A: An unusual three-way translocation $\mathrm{t}(21 ; 8 ; 1)(\mathrm{q} 22 ; \mathrm{q} 22 ; \mathrm{q} 32)$ in a case of acute myeloid leukemia (M2). Ann Biol Clin 70(2): 213216, 2012. PMID: 22484534. DOI: 10.1684/abc.2012.0691

11 Vundinti BR, Kerketta L, Madkaikar M, Jijina F and Ghosh K: Three way translocation in a new variant of $t(8 ; 21)$ acute myeloid leukemia involving Xp22. Indian J Cancer 45(1): 30 32, 2008. PMID: 18453738.

12 Kikuchi A, Hanada R and Yamamoto K: Novel three-way translocation $\mathrm{t}(5 ; 8 ; 21)$ in acute myeloblastic leukemia (M2) with chloroma. J Pediatr Hematol Oncol 21(5): 452-454, 1999. PMID: 10524466.

13 Gallego M, Carroll AJ, Gad GS, Pappo A, Head D, Behm F, Ravindranath Y and Raimondi SC: Variant $\mathrm{t}(8 ; 21)$ rearrangements in acute myeloblastic leukemia of childhood. Cancer Genet Cytogenet 75(2): 139-144, 1994. PMID: 8055478.

14 Ayraud N, Raynaud S, Bayle J and Dujardin P: Variant translocation $\mathrm{t}(8 ; 21 ; 15)$ in an acute myeloblastic leukemia with phenotypic differential evolution. Cancer Genet Cytogenet 15(34): 191-197, 1985. PMID: 3855685.
15 Miyoshi H, Shimizu K, Kozu T, Maseki N, Kaneko Y and Ohki M: $\mathrm{t}(8 ; 21)$ breakpoints on chromosome 21 in acute myeloid leukemia are clustered within a limited region of a single gene, AML1. Proc Natl Acad Sci U S A 88(23): 10431-10434, 1991. PMID: 1720541. DOI: 10.1073/pnas.88.23.10431

16 Barresi V, Romano A, Musso N, Capizzi C, Consoli C, Martelli MP, Palumbo G, Di Raimondo F and Condorelli DF: Broad copy neutral-loss of heterozygosity regions and rare recurring copy number abnormalities in normal karyotype-acute myeloid leukemia genomes. Genes Chromosomes Cancer 49(11): 101410123, 2010. PMID: 20725993. DOI: $10.1002 / \mathrm{gcc} .20810$

17 Xu X, Bryke C, Sukhanova M, Huxley E, Dash DP, DixonMciver A, Fang M, Griepp PT, Hodge JC, Iqbal A, Jeffries S, Kanagal-Shamanna R, Quintero-Rivera F, Shetty S, Slovak ML, Yenamandra A, Lennon PA and Raca G: Assessing copy number abnormalities and copy-neutral loss-of-heterozygosity across the genome as best practice in diagnostic evaluation of acute myeloid leukemia: An evidence-based review from the cancer genomics consortium (CGC) myeloid neoplasms working group. Cancer Genet 228-9: 218-235, 2018. PMID: 30344013. DOI: 10.1016/j.cancergen.2018.07.005

18 Stagno F, Vigneri P, Del FabroV, Stella S, Cupri A, Massimino M, Consoli C, Tambè L, Consoli ML, Antolino A and Di Raimondo F: Influence of complex variant chromosomal translocations in chronic myeloid leukemia patients treated with tyrosine kinase inhibitors. Acta Oncol 49(4): 506-508, 2010. PMID: 20331405. DOI: 0.3109/02841861003660031

19 Stagno F, Vigneri P, Consoli ML, Cupri A, Stella S, Tambè L, Massimino M, Manzella L and Di Raimondo F: Hyperdiploidy associated with a high BCR-ABL transcript level may identify patients at risk of progression in chronic myeloid leukemia. Acta Haematol 127(1): 7-9, 2012. PMID: 21986290. DOI: 10.1159/ 000330607 